\title{
EFFET D'UNE ALIMENTATION PRÉCOCE OU RETARDÉE SUR LA CROISSANCE D'ALEVINS DE TRUITE ARC-EN-CIEL (Salmo gairdneri) ISSUS D'GUFS DE TAILLES DIFFÉRENTES
}

\author{
Anne-Marie ESCAFFRE, P. BERGOT \\ I.N.R.A. Centre de Recherches Hydrobiologiques \\ Laboratoire de Nutrition et d'Élevage des poissons \\ Saint-Pée-sur-Nivelle - 64310 ASCAIN, France.
}

\begin{abstract}
RESUME
-La croissance d'alevins de truite arc-en-ciel est étudiée pendant les 58 jours qui suivent l'éclosion. Les alevins comparés sont des animaux plein-frères, élevés à une temperature moyenne de $10,3^{\circ} \mathrm{C}$. Ils sont issus de lots d'œufs de tailles différentes séparés par un tri mécanique au stade œillé et sont nourris soit dès l'ouverture de l'œsophage, soit 9, 14 et 22 jours plus tard selon les lots. Les alevins atteignent un poids final d'autant plus grand qu'ils proviennent d'œufs plus gros et qu'ils sont nourris plus tôt. Le poids des alevins à 58 jours apparaît lié au poids initial (au moment du premier repas) et au nombre de jours d'alimentation. Le taux de croissance spécifique des différents lots nourris ne paraît influencé ni par la taille de l'œuf ni par le retard d'alimentation. Pendant la période étudiée les gros alevins gardent leur avantage initial sur les petits et les alevins nourris plus tôt conservent leur avance sur ceux qui sont nourris plus tard.
\end{abstract}

\section{INFLUENCE OF EARLY OR DELAYED FEEDING ON GROWTH OF RAINBOW TROUT ALEVINS (Salmo gairdneri) ORIGINATING FROM EGGS OF DIFFERENT SIZES.}

\section{SUMMARY}

Growth of rainbow trout alevins is studied during the 58 days following hatching. The compared alevins are full sib fish, reared under a mean temperature of $10.3^{\circ} \mathrm{C}$. They are originating from eggs of different sizes, mechanically sorted out at the eyed stage, and they are fed either from the oesophagus opening or 9,14 and 22 days later, according to fish groups. The final weight of alevins is as much higher as they are originating from bigger eggs and as they are fed earlier. The weight of alevins when they are 58 days old appears to be related with their initial weight (at the time of first feeding) and with the number of feeding days. It seems that the rate of specific growth in the different fed groups is not influenced either by egg size or by delayed feeding. During the period of time under study, big alevins keep their first advantage over small ones and alevins fed earlier maintain their advance over those fed later.

\section{INTRODUCTION}

Les alevins de Salmonidés possèdent encore des réserves vitellines lorsque le bouchon de cellules épithéliales qui obstruait leur œsophage disparaît, et qu'ils deviennent capables d'ingérer de la nourriture exogène (EGOUNOFF, 1907 ; TWONGO et MacCRIMMON, 1977). En l'absence de tout apport alimentaire l'utilisation du vitellus permet aux alevins de poursuivre leur croissance jusqu'à ce que leur poids corporel atteigne un maximum (GRAY, 1926). Ce maximum est plus élevé et il est atteint plus tardivement à basse température qu'à haute température (GRAY, 1928 ; HEMING et al., 1982 ; HEMING, 1982 ; WALLACE et AASJORD, 1984b). Il est également plus élevé et légèrement plus tardif chez les alevins issus de gros œufs que chez les alevins issus de petit œufs (ESCAFFRE et BERGOT, 1984). Après ce maximum le poids corporel des alevins diminue et la reprise de la croissance nécessite un apport de nourriture exogène. Plusieurs auteurs ont recherché à quel stade il était préférable de commencer à alimenter les alevins. Certains préconisent une alimentation précoce, dès le gonflement de la vessie natatoire (PALMER et al., 1951). Pour d'autres, il est inutile de nourrir avant le stade "poids maximum de l'alevin vésiculé" (MARR, 1965), ou même avant la résorption complète du vitellus (HURLEY et BRANNON, 1969 ; MacCRIMMON et TWONGO, 1980). Pour HEMING et al. (1982), il existe après l'ouverture de l'œsophage une période pendant laquelle l'ingestion de nourriture ne procure pas de croissance supplémentaire par rapport à la seule utilisation du vitellus. Au delà de cette période définie par les auteurs en fonction de la température d'élevage, un retard d'alimentation entraîne une diminution du poids ultérieur des alevins. Exprimée en degrés-jours la période d'insensibilité au retard d'alimentation apparaît beaucoup plus courte à $12^{\circ} \mathrm{C}$ qu'à $6^{\circ} \mathrm{C}$. On peut tout efois se 
demander si la différence est seulement due à la température car les alevins comparés dans cette période ont un poids plus élevé à $6^{\circ} \mathrm{C}$ qu’à $12^{\circ} \mathrm{C}$.

Pour préciser les facteurs qui déterminent la croissance des alevins de truite, il a paru intéressant de comparer, à la même température, l'évolution du poids corporel des animaux ayant initialement des tailles différentes et nourris de façon précoce ou tardive.

\section{MATERIEL ET METHODES}

\section{Matériel animal}

\section{a. Constitution des classes d'œufs}

La ponte d'une femelle truite arc-en-ciel de 3 ans présentant des œufs de taille hétérogène est fécondée par le sperme d'un seul mâle. La fécondation est pratiquée selon la méthode de PETIT et al. (1973) en utilisant le dilueur "532" (BILLARD, 1977). Les œufs sont incubés à une température constante de $7^{\circ} \mathrm{C}$ dans la pisciculture de LEES-ATHAS (Pyrénées Atlantiques). Au stade "œillé", lés œufs sont transportés dans la pisciculture de ST PEE-SUR-NIVELLE (Pyrénées Atlantiques) où l'expérience a lieu dans les bacs de la salle d'élevage (longueur : $40 \mathrm{~cm} ;$ largeur : $20 \mathrm{~cm}$; hauteur d'eau : $10 \mathrm{~cm}$ ) à une température moyenne de $10,3^{\circ} \mathrm{C}$ (écarts maxi $9,1^{\circ} \mathrm{C}-11,5^{\circ} \mathrm{C}$ ).

Les œufs morts (œufs blancs) qui représentent 16 p. 100 de la ponte sont éliminés puis les œufs vivants sont triés par classe de diamètre. Trois trieurs constitués de barres de verre parallèles espacées de 3,7 ou 4 ou $4,2 \mathrm{~mm}$ sont employés successivement. La méthode de triage consiste a déverser délicatement les œufs "œillés" contenus dans un récipient d'eau à la température d'élevage dans un autre récipient d'eau au fond duquel est placé un trieur. Les œufs se déposent alors sur les barres de verre et le triage est facilité par une agitation douce et horizontale du trieur. Cette manipulation est répétée pour obtenir 4 classes d'œufs dont 3 sont retenues dans cette expérience (tableau 1).

\begin{tabular}{|c|c|c|c|}
\hline classes & $\begin{array}{l}\text { Diamètre } \\
\text { des oeufs }\end{array}$ & Nombre d'oeufs & $\begin{array}{l}\text { Poids moyen d'un oeuf } \\
\mathrm{m} \pm \mathrm{SE}(\mathrm{n}=30)\end{array}$ \\
\hline 1 & $<3,7 \mathrm{~mm}$ & 373 & $30,8 \pm 1,2 \mathrm{mg}$ \\
2 & $3,7 \mathrm{a} 4 \mathrm{~mm}$ & 330 & $37,1 \pm 0,6 \mathrm{mg}$ \\
- & 4 à $4,2 \mathrm{~mm}$ & 1633 & $52,9 \pm 1,1 \mathrm{mg}$ \\
3 & $>4,2 \mathrm{~mm}$ & 750 & $61,3 \pm 0,9 \mathrm{mg}$ \\
\hline
\end{tabular}

Tableau 1 : Caractéristiques des ceufs cillés au moment du tri 17 jours avant le début de l'éclosion).

Table 1 : Characteristics of eyed eggs at sorting (7 days before start of hatching).

30 œufs sont prélevés au hasard dans chaque classe puis pesés séparément pour établir un histogramme de fréquence des poids (figure 1). La variabilité du poids des œufs est estimée à 23 p. 100 . Les œufs étant de forme ellipsoïde, c'est en principe leur plus petit diamètre qui est sélectionné. Ce fait peut expliquer le recoupement partiel et la relative symétrie des sous populations obtenues par tri.

\section{b. Constitution des lots d'alevins et prélèvements d'échantillons}

Après le tri, les œufs "œillés" de chaque classe sont répartis dans les bacs par nombre de 300 à 350. L'éclosion commence 7 jours après le tri. Elle débute au cours de la même journée pour les 3 classes et s'étend sur 3 jours quelle que soit la classe de taille d'œuf. Les alevins éclos sont gardés à jeun. Quelques-uns de ces alevins sont prélevés quotidiennement dans les 3 classes pour être disséqués afin de déterminer leur stade de développement.

Trois stades sont retenus: l'ouverture de l'cesophage, la résorption du vitellus et le seuil 50 p. 100 de mortalité chez les alevins gardés à jeun. 16 jours après la fin de l'éclosion, la majorité des alevins des trois classes atteignent le stade "ouverture de l'cesophage" qui correspond à l'apparition de la bulle d'air dans la vessie natatoire. Cette bulle d'air est captée par l'alevin à la surface de l'eau pour obtenir son équilibre de nage (TAIT, $1960 ;$ HELAND, 1977). A ce stade il est fréquent qu'une ou plusieurs bulles d'air soient également visibles dans l'intestin. Au stade "résorption du vitellus" un reste du syncytium vitellin est encore présent mais on n'observe plus de vitellus. 


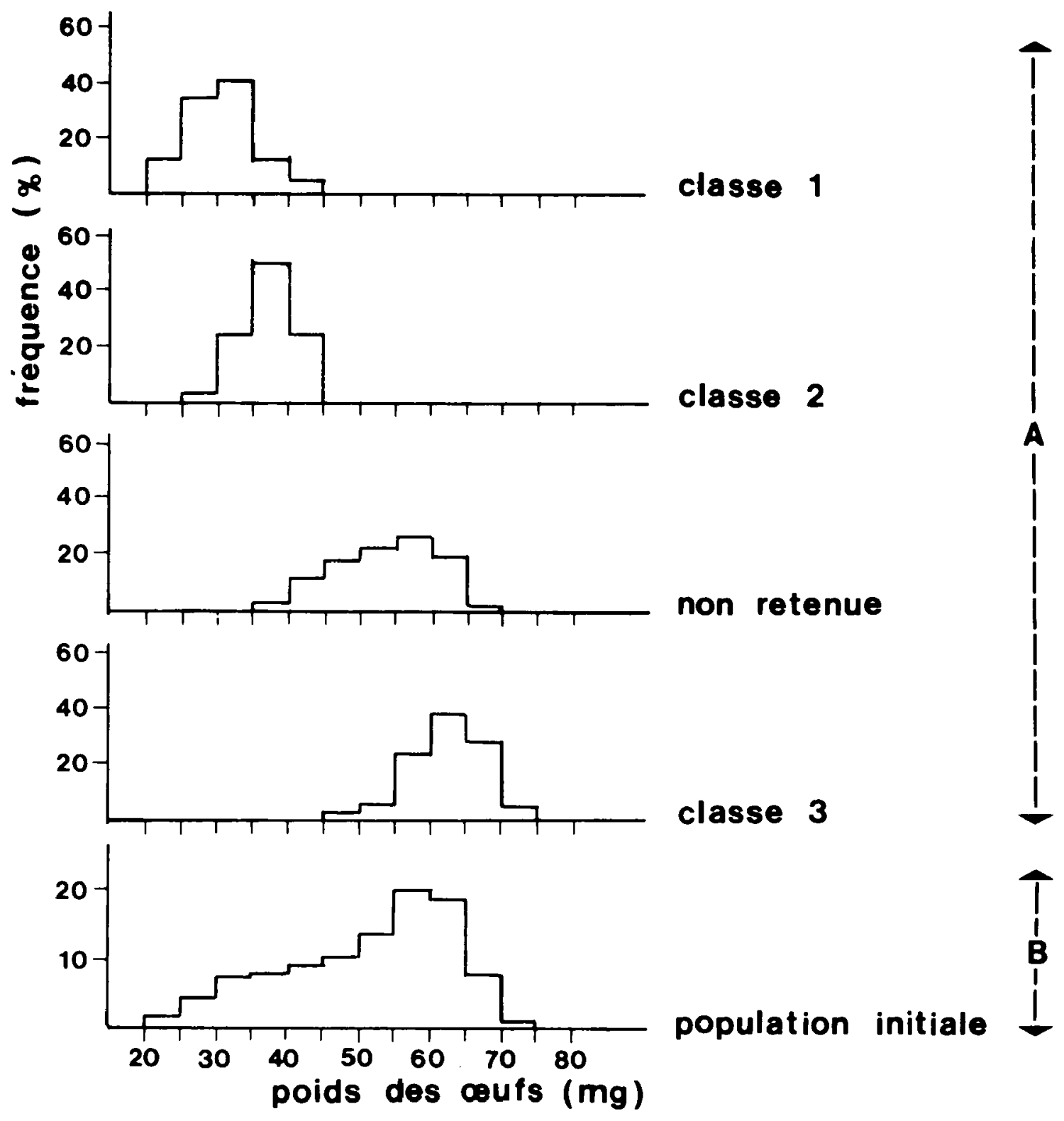

Figure 1 : : Histogrammes de fréquence du poids des coufs.

A) Fréquence observée dans chaque classe (échantillon de 30 coufs)

B) Fréquence estimée pour la population de départ d'aprés les effectifs de chaque classe.

Figure 1: Frequency distribution of egg weight.

A) Observed frequency in each class (sample size : 30 eggs)

B) Estimated frequency in total population, based on observed frequencies in each class.

Le tableau Il donne le moment d'apparition des stades de développement par rapport à la fin de l'éclosion chez les alevins provenant des 3 lots gardés à jeun (lots A) et la mise en place des lots d'alevins à nourrir (lots B, C, D, E) en fonction de ces stades. Sur ce tableau figurent aussi les prélèvements d'échantillons effectués dans chaque lot pour la présente étude et pour une étude histologique ultérieure.

\section{c. Alimentation des alevins}

Durant le premier mois d'expérience, un aliment commercial (démarrage alevin Fario, AQUALIM) est distribué en excès quatre fois par jour aux alevins des lots nourris. Lors du 2 ème mois, un 


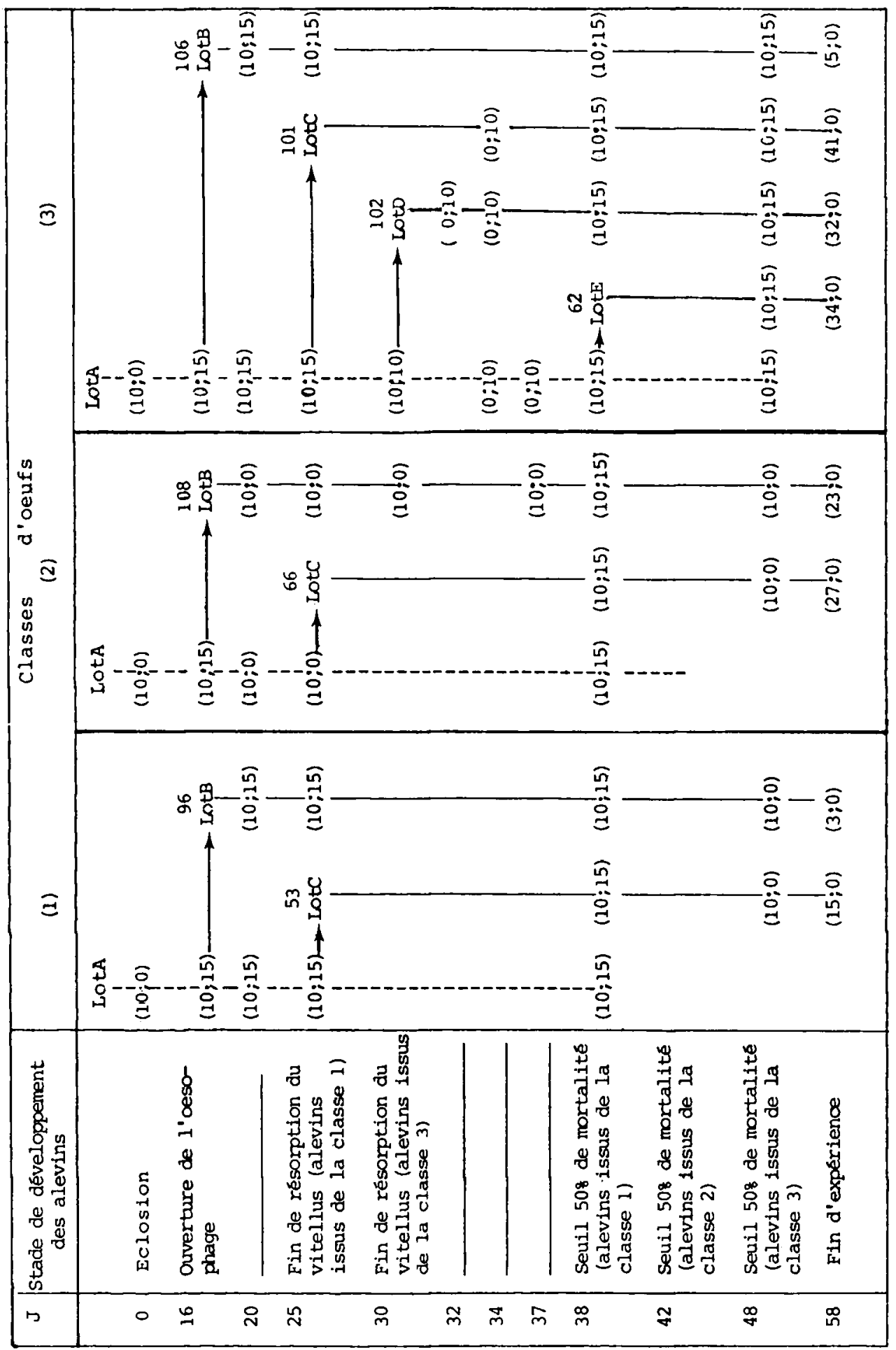

Tableau II : Stades de développement des alevins, constitution des lots et effectifs prélevés (J, nombre de jours après la fin de l'éclosion : - - - lots a jeun ; _ lots nourris : $\longrightarrow n$, nombre initial d'individus dans le lot à nourrir $;\left(n_{1} ; n_{2}\right)$, nombre d'individus prélevés : $n_{1}$ pour l'étude de croissance et $n_{2}$ pour une étude histologique ultérieure.

Table II : Development stages of alevins, constitution of groups and number of samples ( $J$, number of days after the end of hatching : - - - fasted groups : _- fed groups : $\longrightarrow$, initial number of animals in fed group $:\left(n_{1} ; n_{2}\right)$. number of fish sampled : $n_{1}$ for growth studies and $n_{2}$ for later histological observations. 
mélange d'aliments commerciaux (2/3 démarrage alevin Fario, $1 / 3$ 1er âge Fario AQUALIM) est distribué dans les mêmes conditions.

Une observation qualitative de la prise de nourriture est faite après le stade "ouverture de l'œsophage" dans les lots B. La plupart des animaux des 3 classes nourris dès l'apparition de la bulle d'air dans la vessie natatoire possède des particules alimentaires dans leur tractus digestif 24 heures après leur premier repas ; la nourriture ingérée, appréciée qualitativement, est peu abondante 4 jours après le stade "ouverture de l'œsophage", mais au 9ème jour, elle est très abondante et provoque un gonflement du tractus digestif de tous les alevins prélevés dans les 3 classes.

La mortalité est enregistrée quotidiennement dans chaque lot.

\section{Méthodes}

Les alevins prélevés sont sacrifiés par anesthésie profonde au phénoxy-éthanol, puis ceux utilisés dans le présent travail sont conservés dans une solution de formol à 10 p. 100. Après 1 mois de conservation, le corps des alevins est pesé avec une précision de $\pm 0.1 \mathrm{mg}$. Chez les alevins encore vésiculés, le corps est dissocié du reste du vitellus et ces deux parties sont pesées séparément pour chaque individu.

Les données relatives aux mortalités, aux poids du corps et du vitellus sont traitées par les méthodes d'analyses statistiques classiques $\chi^{2}$, test $t$, analyse de la variance, régression) décrites par SNEDECOR et COCHRAN (1971). Dans cette étude, les moyennes sont données suivies de leur écart type (SE).

\section{RÉSULTATS}

\section{Résorption du vitellus et survie des alevins}

Dans les lots A (animaux gardés à jeun) la quantité de vitellus, en valeur absolue et relative, est plus importante chez les alevins issus de gros œufs que chez ceux issus de petits œufs, en fin d'éclosion (J0), à l'ouverture de l'œsophage (J16) et à J2O (tableau III).

\begin{tabular}{|c|c|c|c|c|c|c|}
\hline \multirow{2}{*}{ V1tellus } & \multirow{2}{*}{$\begin{array}{c}\text { tot } A \\
\text { classes }\end{array}$} & \multicolumn{5}{|c|}{ Nombre de jours après la fin de l'éclosion } \\
\hline & & 0 & 16 & 20 & 25 & 30 \\
\hline \multirow{3}{*}{$\begin{array}{l}\text { Poids absolu } \\
\text { en mg }\end{array}$} & 1 & $12,47 \pm 0,88$ & $1,77 \pm 0,30$ & $0,37 \pm 0,03$ & 0 & - \\
\hline & 2 & $16,3 \pm 0,53$ & $3,02 \pm 0,47$ & $0,63 \pm 0,22$ & $0,02 \pm 0,02$ & - \\
\hline & 3 & $29,07 \pm 0,82$ & $5,16 \pm 0,41$ & $1,66 \pm 0,37$ & $0,07 \pm 0,05$ & 0 \\
\hline \multirow{3}{*}{$\begin{array}{l}\text { Poids relatif en } \\
\text { du poids de } \\
\text { 'alevin entier } \\
\text { (corps + vitelius) }\end{array}$} & 1 & $56,34 \pm 1,08$ & $4,59 \pm 0,68$ & $0,78 \pm 0,29$ & & \\
\hline & 2 & $62,45 \pm 2,15$ & $5,50 \pm 0,77$ & $1.25 \pm 0.49$ & & \\
\hline & 3 & $62,50 \pm 0,83$ & $6,46 \pm 0,53$ & $1,77 \pm 0,40$ & & \\
\hline $\begin{array}{l}\text { Comparaison du poids } \\
\text { relatif des classes } \\
\text { extrames (1 et } 3)\end{array}$ & & $P<1$ p. 1000 & $P<1$ P. 100 & $P<1 p .200$ & & \\
\hline
\end{tabular}

Tableau III : Résorption du vitellus dans les 3 lots d'alevins gardés à jeun (moyenne de 10 individus \pm SE)

Table III : Yolk resorption in the 3 groups of fasted alevins (means of 10 animals \pm SE)

La durée de résorption du vitellus est différente entre les classes extrêmes (tableau III). Tous les alevins examinés dans la classe 1 (petits alevins) n'ont plus de vitellus à partir du 25 ème jour qui suit la fin de l'éclosion alors qu'il faut attendre le 30 ème jour pour les alevins de la classe 3 (gros œufs).

La mortalité entre le tri et la fin de l'éclosion est très faible. Elle est de $0,6 \mathrm{p}$. 100 dans la classe 1, 0,3 p. 100 dans la classe 2 et 0,1 p. 100 dans la classe 3 . Après la fin de l'éclosion, l'évolution de la mortalité des alevins dans les lots gardés à jeun et nourris est représentée sur la figure 2.

Dans les lots qui ne reçoivent pas de nourriture (lots $A$ ), les gros alevins résistent plus longtemps au jeûne que les petits. Le stade "50 p. 100 de mortalité" est atteint respectivement à J38, J42 et J48 pour les classes 1,2 et 3. 


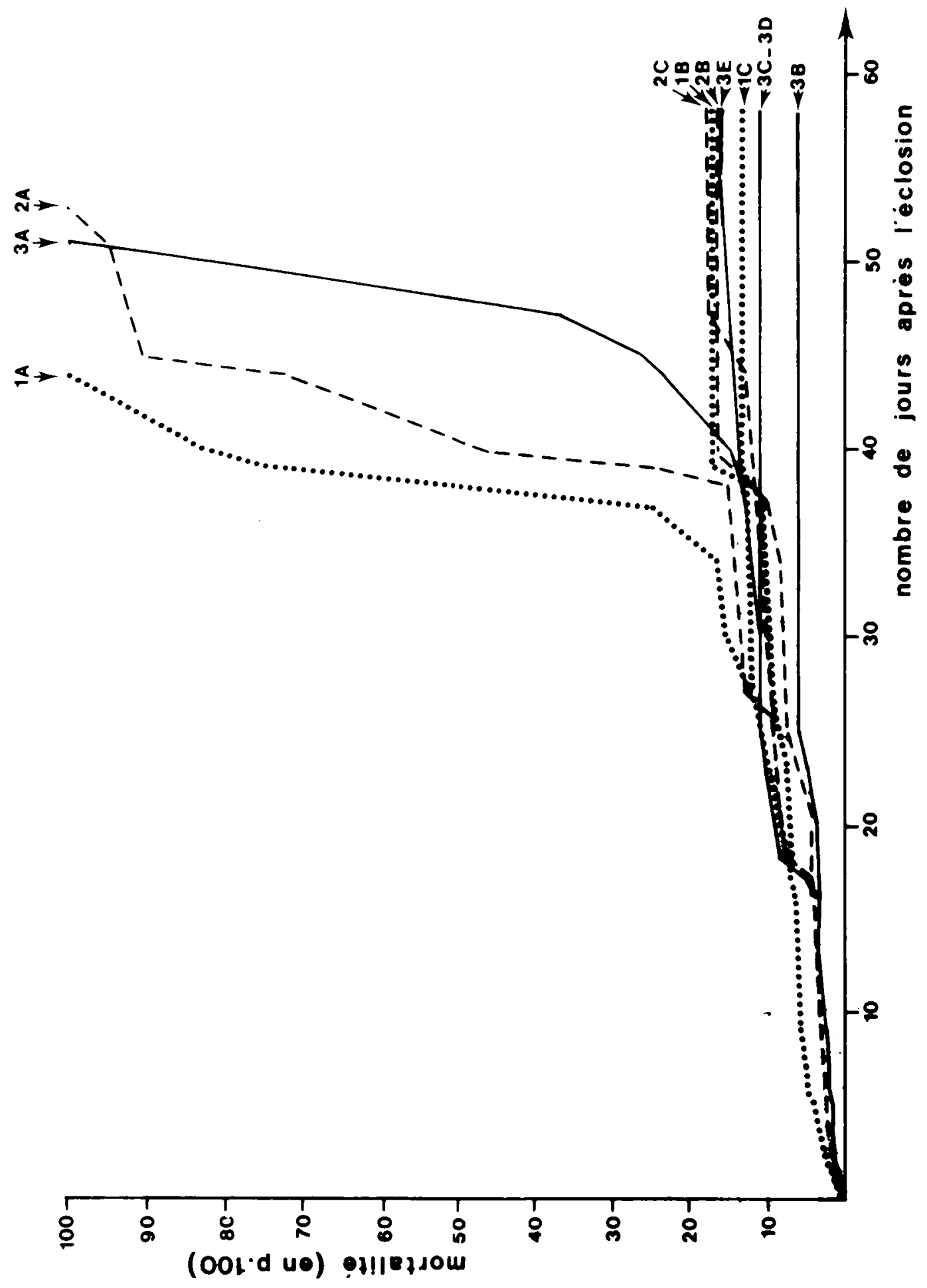

Figure 2 : Evolution de la mortalité des alevins dans les 11 lots expérimentaux (...... classe 1 ; -. - classe 2 ; classe 3 ).

Figure 2: Evolution of alevins mortality in the 11 experimental groups (..... class 1 ; _. - class 2 : - class 3 ). 
Dans les lots nourris, les taux de mortalité relevés en fin d'expérience ne sont pas significativement différents $\left(\chi^{2}=10.6 ; 7 \mathrm{dl}\right.$; NS). A J58, dans les lots nourris précocement (lots B) on enregistre une mortalité comprise entre 6 et 17 p. 100 et dans les lots nourris tardivement (lots C. D. E) entre 11 et 18 p. 100 .

\section{Croissance pondérale (figure 3)}

A l'éclosion, le poids du corps des alevins varie en fonction de la grosseur de l'ceuf d'origine. Les alevins issus des plus petits œufs (classe 1 ) ont un poids moyen de 11,9 $\pm 0,7 \mathrm{mg}$ ( $\pm \mathrm{SE}$ ) et ceux issus des plus gros œufs (classe 3 ) de 19,5 $\pm 0,5 \mathrm{mg}$ (土 SE).

Chez les alevins gardés à jeun (lots A), le poids corporel augmente après l'éclosion. Il atteint une valeur maximum vers $\mathrm{J} 20$ pour les alevins issus des petits œufs et vers $\mathrm{J} 25$ pour ceux issus des gros œufs. Cette valeur maximum est située entre les stades "ouverture de l'œesophage" et "résorption du vitellus". Elle est environ deux fois plus élevée dans la classe 3 que dans la classe 1 . Après ce maximum, le poids corporel des alevins diminue dans les 3 classes et se différencie significativement du maximum au stade "50 p. 100 dermortalité".

Chez les alevins nourris précocement (lots B), 58 jours après l'éclosion, on note une différence significative $(P<0,01)$ entre les poids corporels des animaux des 3 classes. Le poids moyen des alevins issus des petits œufs (classe 1) représente $56 \mathrm{p}$. 100 du poids de ceux issus des gros œufs (classe 3 ). Ce rapport est proche de celui observé entre ces deux classes à l'éclosion (61 p. 100).

Chez les alevins de la classe 3 nourris tardivement, on observe 58 jours après l'éclosion, un poids corporel d'autant plus faible que le retard d'alimentation est plus grand. A l'intérieur de chaque classe, le poids moven des animaux nourris à partir du 9ème jour qui suit l'ouverture de l'œesophage (lots C) est plus faible que celui des animaux nourris dès l'ouverture de l'œsophage (lots $B$ ).

Dans chaque lot, le poids du corps des alevins nourris augmente en fonction du nombre de jours d'alimentation. A la vue des courbes de la figure 3, un modèle simple de croissance de type exponentiel $\left(P=P_{o} e^{k t}\right)$, est utilisé pour calculer le taux de croissance des alevins nourris $\left(P_{o}=\right.$ poids du corps au moment du premier repas $; t=$ nombre de jours d'alimentation $; k=$ taux de croissance spécifique).

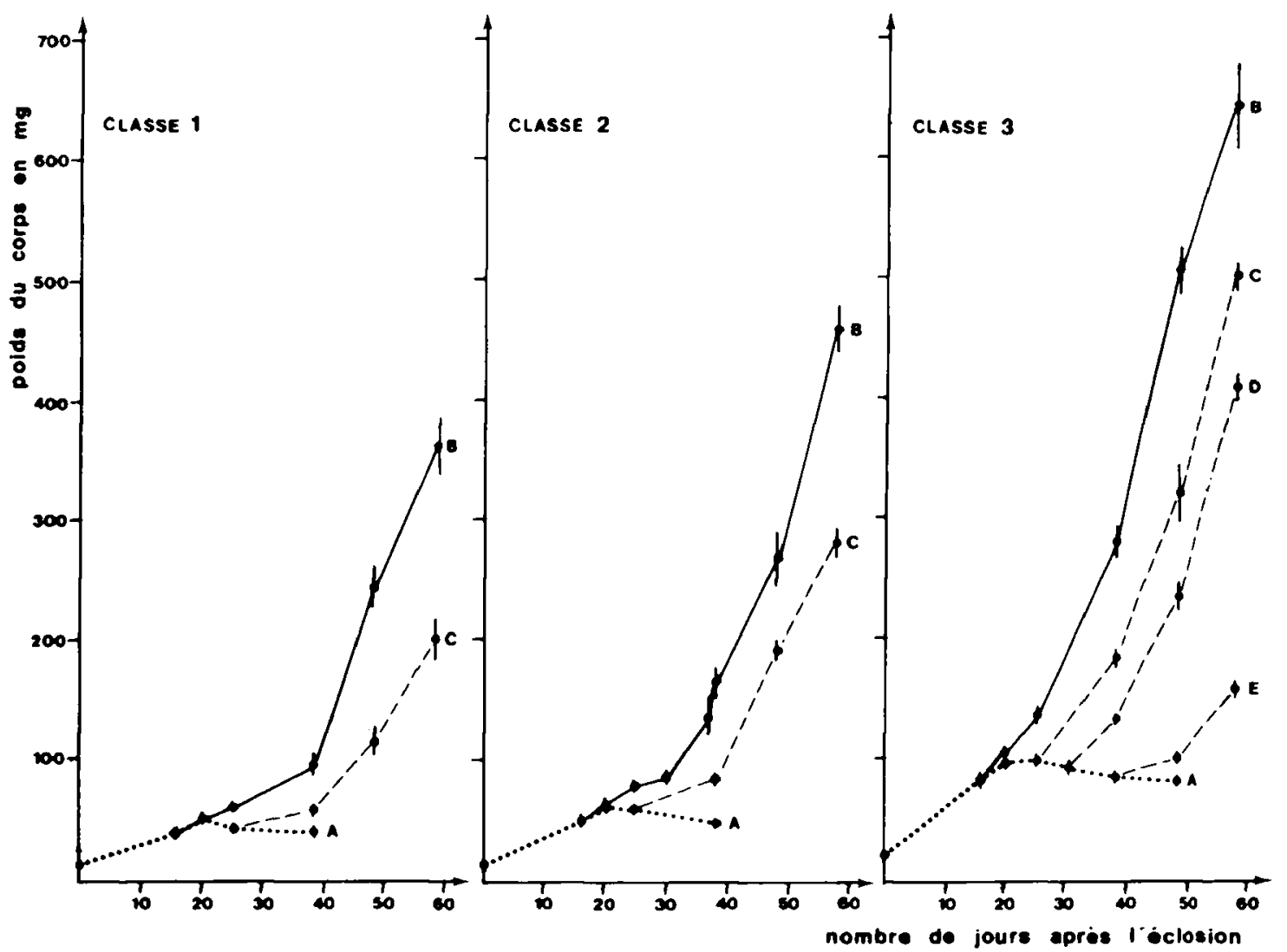

Figure 3 : Evolution du poids corporel des alevins dans les 11 lots expérimentaux (....... lots gardés à jeun :- lots nourris précocement :- - - lots nourris tardivement).

Figure 3 : Evolution of body weight of alevins in the 11 experimental groups $(\ldots . .$. fasted groups :-_ early fed groups : - - - late fed groups). 
Le calcul des régressions est effectué à partir de la relation LnP $=$ LnPo $+k t$ et les caractéristiques de ces régressions sont données dans le tableau IV. Comme tous les coefficients de corrélations linéaires sont élevés, on a négligé les écarts à la linéarité, significatifs dans certains lots, mais très faibles. Le modèle utilisé donne une meilleure approximation de la croissance du poids corporel des alevins issus des gros œufs que de celle des alevins issus des petits œufs, les carrés moyens réduits augmentant inversement à la taille de l'œuf d'origine.

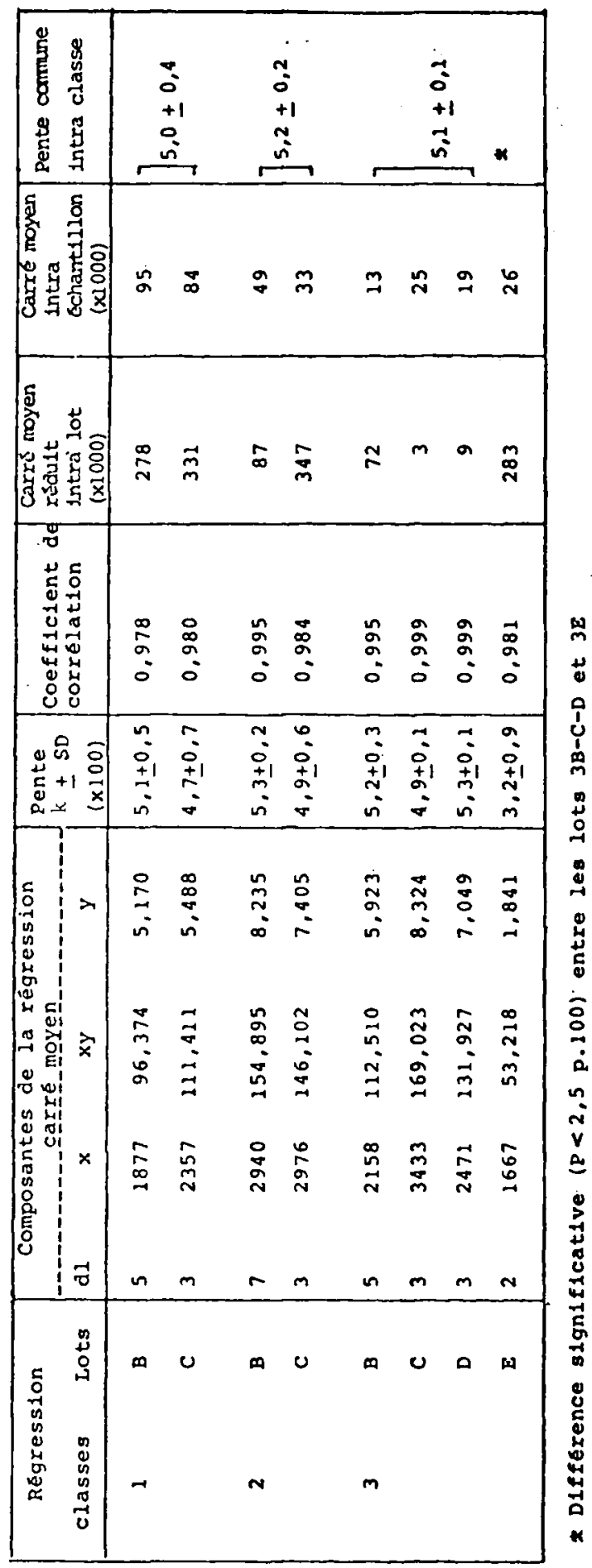

Tableau IV : Régressions des lots d'alevins nourris. $X=$ nombre de jours d'alimentation : $Y=$ Ln du poids corporel.

Table IV: Regressions in fed groups. $X=$ number of days of feeding : $Y=$ Ln of body weight. 
Jusqu'au stade "résorption du vitellus", le retard d'alimentation n'affecte pas le taux de croissance spécifique (pente de la régression linéaire) et un taux moyen peut être calculé pour chaque classe (tableau IV). Au delà du stade "résorption du vitellus", le retard d'alimentation de 8 jours (lot 3E) par contre fait diminuer significativement la pente (figure 4). Les pentes moyennes des 3 classes étant semblables, le taux de croissance spécifique commun à tous les alevins nourris, excepté ceux du lot $3 E$, a pour valeur $5,1 \pm 0,1$ p. 100

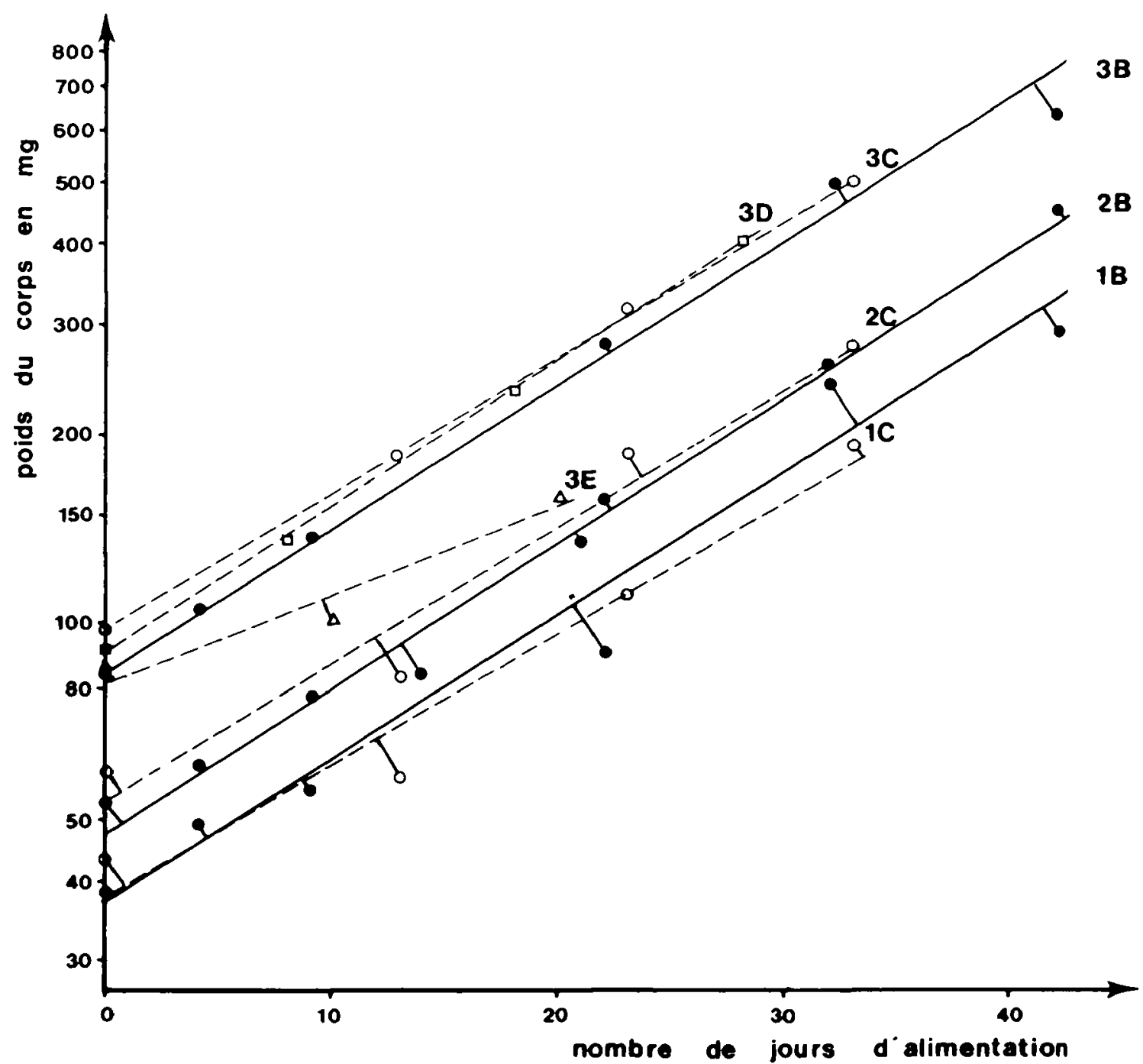

Figure 4: Régression du poids corporel des alevins sur le nombre de jours d’alimentation dans les 8 lots nourris : lots nourris précocement :- - - lots nourris tardivement. (début d'alimentation après l'ouverture de l'cesophage : 0 jour, 09 jours. $\square 14$ jours, $\triangle 22$ jours).

Figure 4: Regression of alevin body weight upon number of days of feeding in the 8 fed groups : _- early fed groups :- - - late fed groups. (delay between cesophagus opening and beginning of feeding : 0 day, $\bigcirc 9$ days, $\square 14$ days, $\Delta 22$ days).

\section{DISCUSSION}

Comme dans un travail précédent (ESCAFFRE et BERGOT, 1984) il a été possible de constituer à partir d'une ponte hétérogène des classes d'œufs de poids moyens notablement différents, l'écart entre les classes extrêmes étant du simple au double. L'utilisation d'animaux plein-frères présente des inconvénients (effectifs relativement faibles des classes comparées et nécessité de recourir à des femelles dont la variabilité de la taille des œufs est très élevée, ce qui peut dénoter un caractère 
atypique) mais aussi des avantages par rapport à la comparaison de pontes de femelles d'âge différent (KAMLER et KATO, 1983) ou de classes d'alevins constituées à partir d'une population d'œufs provenant de géniteurs non identifiés (WALLACE et AASJORD, 1984a). En effet, les variations de survie et de croissance d'origine génétique (GALL, 1974 ; KANIS et al., 1976 ; PITMAN, 1979) sont réduites et les conclusions sur l'effet de la taille de l'œuf sont plus sûres

Les mortalités observées immédiatement après le tri et en cours d'expérience sont faibles. Cela permet de penser que le tri est une opération peu traumatisante pour les œufs et que les alevins étudiés, bien que représentant les extrêmes de la distribution de tailles, ont des caractéristiques normales de développement. D'après BILTON et ROBINS (1973), le retard d'alimentation jusqu'à la résorption du vitellus n'affecte pas le taux de survie des alevins de Salmonidés. Dans cette expérience nous observons que ce fait est valable aussi bien pour les gros alevins que pour les petits.

La taille de l'cuf d'origine ne paraît pas avoir d'influence sur le moment où l'alevin devient apte à se nourrir. Les alevins mangent peu lors des premiers jours qui suivent l'ouverture de l'œesophage. MacCRIMMON et TWONGO (1980) ont montré que les alevins avaient besoin d'une période d'apprentissage pour se nourrir, plus ou moins longue selon la température d'élevage. WALLACE et AASJORD (1984a), chez l'omble chevalier, rapportent que lorsque 100 p. 100 des gros alevins se nourrissent, 50 p. 100 des plus petits alevins ont encore leur tube digestif vide malgré la disponibilité de la nourriture. Ces auteurs pensent que le retard d'alimentation initial des petits alevins expliquerait leur faible taux de croissance par rapport aux gros alevins. Une telle situation n'a pas été rencontrée dans le présent travail où la supériorité de taille des plus gros alevins à un âge donné ne peut être imputée à un nombre plus grand de jours d'alimentation réelle.

Les effectifs des lots comparés varient entre eux. Un effet de la densité sur la croissance pourrait être envisagé. Cependant on peut remarquer que les densités utilisées (au plus 1.350 alevins $\left./ \mathrm{m}^{2}\right)$ sont bien inférieures aux valeurs maximales $\left(10.000\right.$ alevins $/ \mathrm{m}^{2}$ à 1 mois et $3.000 / \mathrm{m}^{2}$ à 2 mois) indiquées par HUET (1970). Pour un lot donné, le poids moyen des alevins augmente en cours d'expérience tandis que leur effectif diminue à la suite des prélèvements, ce qui conduit à une charge maximale par bac après 3 ou 4 semaines d'alimentation. Or on ne constate pas de diminution particulière de la croissance à ce moment-là. En fin d'expérience, les très faibles densités rencontrées dans certains lots pourraient, par suite de phénomènes de compétition, affecter la croissance moyenne du lot. Ces phénomènes apparaissent en effet très précocement (HELAND, 1977). Comme la nourriture est distribuée en excès à chaque repas, on peut penser que les effets de compétitions sont réduits.

L'évolution du poids corporel des animaux gardés à jeun est conforme aux résultats obtenus précédemment chez la truite arc-en-ciel (ESCAFFRE et BERGOT, 1984). Les animaux issus des plus gros œufs atteignent un poids maximum plus élevé, résorbent plus tardivement leur vitellus et résistent plus longtemps au jeùne que les animaux issus des petits œufs.

Dans le modèle simple de croissance $P=P_{0} e^{k t}$, utilisé présentement pour analyser l'évolution du poids des alevins nourris, le coefficient $k$, correspondant au taux de croissance spécifique, ne paraît pas affecté par un retard d'alimentation, du moins quand ce retard n'excède pas la résorption du vitellus. Ce résultat est en accord avec ceux de TWONGO et MacCRIMMON (1976) chez la truite et HEMING et al., (1982) chez le saumon. Par contre il diffère des conclusions de WALLACE et AASJORD (1984b) chez l'omble chevalier. Cependant, ces derniers auteurs appliquent le modèle de croissance exponentielle dès l'éclosion et non à partir du premier repas alors que la croissance des alevins avant leur alimentation, en particulier pour ceux qui sont nourris tardivement (après le poids maximum). s'écarte sensiblement de ce modèle. Il apparaît de ce fait plus justifié de comparer les taux de croissance entre les alevins nourris précocement et tardivement seulement à partir du premier repas $\left(P_{0}\right)$.

Dans notre expérience, la valeur de $k$ paraît également iı j́pendante de la taille de l'œuf d'origine. Il est intéressant de rappeler que dans une étude consacrée à l'utilisation du vitellus chez des alevins non alimentés (ESCAFFRE et BERGOT, 1984) nous avions trouvé, à partir d'un modèle plus complexe ajusté aux données, un taux de croissance spécifique initial identique chez les alevins vésiculés, issus de petits ou gros œufs. En conséquence, il semble que les petits et gros alevins provenant de la même fratrie ont le même potentiel de croissance, au moins pendant les deux premiers mois qui suivent l'éclosion. Au-delà de cette période, le potentiel de croissance des alevins issus de petits et gros œufs intra-femelle demanderait à être précisé. BILTON (1971) a observé que le coefficient de corrélation entre la taille de l'œuf et celle de l'alevin est très élevé à 3 mois mais nul à 9 mois chez le saumon. Pour CHEVASSUS (1976), ce coefficient diminue progressivement et s'annule aux environs de 3 mois chez la truite.

La période comprise entre l'ouverture de l'œsophage et le poids maximum apparaît plus courte chez les petits alevins que chez les gros. La zone d'alimentation optimale définie par HEMING et al., (1982), qui correspond à la période où l'utilisation des réserves vitellines seules donne une croissance équivalente à celle obtenue avec l'aliment, serait de ce fait moins importante chez les petits alevins que chez les gros. 
Dans nos conditions expérimentales, la valeur de $k$ (proche de 5 p. 100) commune à tous les lots nourris excepté le lot $3 E$ correspond à un doublement de poids de l'alevin en 14 jours. Le fait d'avoir un poids deux fois plus élevé au moment du premier repas $\left(P_{0}\right)$ confère donc un avantage équivalant à 14 jours d'alimentation aux gros alevins par rapport aux plus petits.

Les écarts de poids notés en fin d'expérience entre les animaux d'une même classe, nourris précocement ou tardivement, sont essentiellement dus à la différence du nombre de jours d'alimentation. En effet, $P_{0}$ varie, mais relativement peu, en fonction du retard d'alimentation (figures 3 et 4 ). Le fait de nourrir les alevins dès l'ouverture de l'œsophage, qui est un stade facile à reconnaître en pisciculture (il correspond à l'équilibre de nage), assure l'obtention d'animaux de poids donné en un temps minimum.

En conclusion, l'élevage séparé des petits et gros alevins peut présenter un intérêt. Nos résultats suggèrent que la pratique d'une alimentation précoce permettrait de réduire la durée de l'alevinage. Des œufs de tailles distinctes peuvent être obtenus à partir de femelles d'âge, d'origine génétique ou recevant des régimes alimentaires différents. Ces facteurs qui n'interviennent pas dans le présent travail doivent être pris en compte avant de conclure sur l'intérêt d'utiliser de gros œufs en pisciculture.

\section{Remerciements}

Des remerciements sont dus à $\mathrm{M}$. J.J. YANCI pour le soin apporté au nourrissage des animaux et à M. J.M. BLANC pour le prêt de la salle d'élevage et la lecture critique du manuscrit.

\section{BIBLIOGRAPHIE}

BILLARD R., 1977. Utilisation d'un système tris-glycocolle pour tamponner le dilueur d'insémination pour truite. Bull. Franc. Pisc., 264, 102-112.

BILTON H.T., 1971. A hypothesis of alternation of age of return in successive generations of skeena river sockeye salmon (Oncorhynchus nerka). J. Fish. Res. Board Can., 28, 513-516.

BILTON H.T., ROBINS G.L., 1973. The effect of starvation and subsequent feeding on survival and growth of Fulton Channel sockeye salmon fry (Oncorhynchus nerka). J. Fish. Res. Board Can., 30, 1-5.

CHEVASSUS B., 1976. Variabilité et héritabilité des performances de croissance chez la truite arc-enciel (Salmo gairdneri Richardson). Ann. Génét. Sél. anim., 8 (2), 273-283.

EGOUNOFF S., 1907. Développement histologique du tube digestif de la truite. Revue Suisse de Zoologie, 15, 19-80.

ESCAFFRE A.M., BERGOT P., 1984. Utilization of the yolk in rainbow trout alevins (Salmo gairdneri Richardson) : effect of egg size. Reprod. Nutr. Dévelop., 24 (4). 449-460.

GALL G.A.E., 1974. Influence of size of eggs and age of female on hatchability and growth in rainbow trout. Calif. Fish Game, 60, 26-35.

GRAY J., 1926. The growth of fish. I. The relationship between embryo and yolk in Salmo fario. J. Exp. Biol., 4, 215-225

GRAY J., 1928. The growth of fish. III. The effect of temperature on the development of the eggs of Salmo fario. J. Exp. Biol. 6, 125-130.

HELAND M., 1977. Recherches sur l'ontogénèse du comportement territorial chez l'alevin de truite commune (Salmo trutta L.). Thèse 3ème cycle, Université de Rennes. 239 pp.

HEMING T.A., 1982. Effects of temperature on utilization of yolk by chinook salmon /Oncorhynchus tshawytscha) eggs and alevins. Can. J. Fish. Aquat. Sci., 39, 184-190.

HEMING T.A., MacINERNEY J.E., ALDERDICE D.F., 1982. Effect of temperature on initial feeding in alevins of chinook salmon (Oncorhynchus tshawytscha). Can. J. Fish. Aquat. Sci., 39. 1554- 1562

HUET M., 1970. Traité de pisciculture, 4e édition, $C H$. DE WYNGAERT ed., BRUXELLES, 718 p.

HURLEY D.A., BRANNON E.L., 1969. Effect of feeding before and after yolk absorption on the growth of sockeye salmon. Int. Pac. Salmon Fish. Comm. Prog. Rep., 21, 1-19.

KAMLER E., KATO T., 1983. Efficiency of yolk utilization by Salmo gairdneri in relation to incubation temperature and egg size. Pol. Arch. Hydrobiol., 30 (3), 271-306.

KANIS E., REFSTIE T., GJEDREM T., 1976. A genetic analysis of egg, alevin and fry mortality in salmon (Salmo salar) sea trout (Salmo trutta) and rainbow trout (Salmo gairdneri). Aquaculture, 8 , 259-268 
MacCRIMMON H.R., TWONGO T.K., 1980. Ontogeny of feeding behaviour in hatchery-reared rainbow trout, Salmo gairdneri Richardson.Can. J. Zool., 58, 20-26.

MARR D.H.A., 1965. Factors affecting the growth of salmon alevins and their survival and growth during the fry stage. Yearb. Ass. River Auth., 1965, 133-141.

PALMER D.D., JOHNSON H.E., ROBINSON L.A., BURROWS R.E., 1951. The effect of retardation of the initial feeding on the growth and survival of salmon fingerlings. Prog. Fish Cult., 13 (2), 55-62.

PETIT. J., JALABERT B., CHEVASSUS B., BILLARD R., 1973. L'insémination artificielle de la truite (Salmo gairdneri Richardson). I. Effet du taux de dilution, du pH et de la pression osmotique du dilueur sur la fécondation. Ann. Hydrobiol., 4, 201-210.

PITMAN R.W., 1979. Effect of female age and egg size on growth and mortality in rainbow trout. Prog. Fish. Cult., 41, 202-204.

SNEDECOR G.W., COCHRAN W.G., 1971. Méthodes statistiques. 6ème Ed. (franc.), ACTA ed., Paris, $649 \mathrm{pp}$.

TAIT J.S., 1960. The first filling of the swim bladder in salmonoids. Can. J. Zool., 38, 179-187.

TWONGO T.K., MacCRIMMON H.R., 1976. Significance of the timing of initial feeding in hatchery rainbow trout, Salmo gairdneri. J. Fish. Res. Board Can., 33, 1914-1921.

TWONGO T.K., MacCRIMMON H.R., 1977. Histogenesis of the oropharyngeal and cesophageal mucosa as related to early feeding in rainbow trout, Salmo gairdneri Richardson. Can. J. Zool., $55(1), 116-128$.

WALLACE J.C., AASJORD D., 1984a. An investigation of the consequences of egg size for the culture of Arctic charr, Salvelinus alpinus (L.). J. Fish Biol., 24, 427-435.

WALLACE J.C., AASJORD D., 1984b. The initial feeding of Arctic charr (Salvelinus alpinus) alevins at different temperatures and under different feeding regimes. Aquaculture, 38, 19-33. 